\title{
Safety and Effectiveness of Shoulder Arthroplasties in Spain: A Systematic Review
}

\author{
Jorge Arias-de la Torre ${ }^{1,2,3,4, *}$, Xavier Garcia ${ }^{1}$, Kayla Smith ${ }^{1,5}$, Arantxa Romero-Tamarit ${ }^{1}$, \\ Elisa Puigdomenech 1,5, Laura Muñoz-Ortiz 1,5, Jonathan P. Evans 6,7, Vicente Martín 2,3, \\ Antonio J. Molina ${ }^{3}$, Carles Torrens ${ }^{8}$, Miquel Pons-Cabrafiga ${ }^{9}$, Francesc Pallisó ${ }^{10}$, \\ Jose María Valderas ${ }^{6}$ and Mireia Espallargues ${ }^{1,5}$ \\ 1 Agency for Health Quality and Assessment of Catalonia (AQuAS), 08005 Barcelona, Spain; \\ xavier.garciacusco@gencat.cat (X.G.); ksmith@gencat.cat (K.S.); arantxa.romero@gencat.cat (A.R.-T.); \\ epuigdomenech@gencat.cat (E.P.); Imunyoz@gencat.cat (L.M.-O.); mespallargues@gencat.cat (M.E.) \\ 2 CIBER Epidemiology and Public Health (CIBERESP), 28029 Madrid, Spain; vicente.martin@unileon.es \\ Institute of Biomedicine (IBIOMED), University of Leon, 24071 León, Spain; ajmolt@unileon.es \\ King's College London, Institute of Psychiatry, Psychology and Neuroscience (IoPPN), London SE5 8AB, UK \\ Health Services Research on Chronic Patients Network (REDISSEC), 28029 Madrid, Spain \\ 6 Health Services and Policy Research Group, University of Exeter Medical School, Exeter EX1 2LU, UK; \\ J.Evans3@exeter.ac.uk (J.P.E.); J.M.Valderas@exeter.ac.uk (J.M.V.) \\ 7 Royal Devon and Exeter NHS Foundation Trust, Exeter EX2 5DW, UK \\ 8 Hospital del Mar, Department of Orthopaedic Surgery and Trauma, 08003 Barcelona, Spain; \\ ctorrens@parcdesalutmar.cat \\ 9 Department of Orthopaedic Surgery and Trauma, Sant Rafael University Hospital, 08035 Barcelona, Spain; \\ 23655mpc@gmail.com \\ 10 Department of Orthopaedic Surgery and Trauma, Santa María University Hospital, 25198 Lleida, Spain; \\ fpalliso@gss.scs.es \\ * Correspondence: jorge.arias_de_la_torre@kcl.ac.uk; Tel.: +34-634-722-677
}

Received: 8 October 2019; Accepted: 21 November 2019; Published: 23 November 2019

\begin{abstract}
The effectiveness and safety of shoulder arthroplasties in the general context of a Spanish patient population remains unclear. The aim of this study was to ascertain both the effectiveness and safety of primary shoulder arthroplasties and the prosthesis types used in Spain. A systematic review of all the available literature evaluating the effectiveness and safety of primary shoulder arthroplasties in Spain was performed. A narrative synthesis was performed, and evidence tables were created in four dimensions: study design, arthroplasty characteristics, safety, and effectiveness. Orthopaedic Data Evaluation Panel (ODEP) scores were used to evaluate prosthesis types. Twenty-one studies were selected that included a total of 1293 arthroplasties. The most common indication was fractures, while the prosthesis most frequently used was the Delta Xtend (ODEP 10A). The most common complication was scapular notching. Prosthesis revision rate was approximately $6 \%$ for follow-ups between 12 and 79 months. In addition, significant improvements were observed in the Constant-Murley test score after the intervention. Currently in Spain, shoulder arthroplasty can be considered a safe and effective procedure with functional recovery and pain reduction for eligible patients with humeral fracture, rotator cuff arthropathy, fracture sequelae and malunion of the proximal humerus, and degenerative disease. Future longitudinal research and population-based studies could serve to confirm these results and identify points of improvement.
\end{abstract}

Keywords: shoulder; arthroplasty; safety; effectiveness; systematic review 


\section{Introduction}

Shoulder arthroplasty is currently considered to be an established therapeutic option and an effective and efficient procedure to improve physical function, pain and quality of life in patients [1-5]. As a result of continual technological progress and emerging indications for shoulder arthroplasty, including proximal humeral fractures, osteoarthritis and massive rotator cuff tears [6], the utilization of this procedure has increased throughout the world and in some countries it has tripled in the last decade [7-9].

The use of shoulder arthroplasty has increased significantly in Spain in recent years [10,11], but remains a lower-volume procedure compared to knee and hip replacement. Given the increasing indication for these types of procedures and their potential to improve the health of patients, it is vitally important for both patients and clinicians that the complications associated with these interventions are well understood. Previous research has proposed that the three most common complications are instability, periprosthetic fracture and infection [12-14]. Understanding this information within the context of the effectiveness of different types of prostheses and models, and in certain population groups, is likely to be highly relevant $[1,7,14]$. However, as far as we know, no studies have evaluated the effectiveness and safety of these procedures in the general context of a Spanish population. Furthermore, there is no arthroplasty register that can be directly assessed. Both analysing the results of these procedures by population and establishing a registry could be useful in evaluating the results of shoulder arthroplasties more precisely in a specific healthcare context such as Spain, facilitating a comparison to other international contexts.

Regarding the safety and effectiveness of primary shoulder arthroplasties, evidence suggests that some of the most frequent complications associated with this procedure are scapular notching, dislocation, periprosthetic fracture, and infection [14-16]. Furthermore, the revision rate of shoulder arthroplasties is estimated to be approximately $5 \%$ and $10 \%$ at 5 and 10 years respectively, and may be lower in reverse arthroplasties compared to hemiarthroplasty and total shoulder arthroplasty [15-17]. However, in the Spanish context, to date no population-level studies have been conducted to evaluate these outcomes, or aimed to quantify the safety and effectiveness of primary shoulder arthroplasties. Furthermore, the evidence found in specific studies that have already been carried out is divergent, which may be due to a focus on specific models or types of prostheses, or certain pathologies and clinical populations [18-20].

In this context, the objective of this study was to describe the scientific evidence available on the effectiveness and safety of primary shoulder arthroplasties in Spain and the types of prosthesis used in this population.

\section{Materials and Methods}

A systematic review was conducted on the results of shoulder arthroplasties performed in public hospitals in Spain, and the results are reported according to the Preferred Reporting Items for Systematic Reviews and Meta-Analyses (PRISMA) criteria [21]. The review has been registered in PROSPERO (CRD42019115342). The following databases were used as sources of information: EMBASE, PubMed, Scielo, Cochrane Reviews and Center for Reviews and Dissemination. The search range was restricted from January 2003 to December 2018. Given the continuous improvement in shoulder arthroplasty results and the advancement in surgical techniques, the lower limit was set at 2003. This limit was fixed in order to maximize the study period and overcome any potential limitations related to underestimating the current results after pooling long-term retrospective data.

A search filter was developed specifically designed for PubMed/Medline to achieve the objectives of this study (Appendix A), and was adapted to other databases. The search strategy was based on previous studies in an attempt to maximize the number of documents identified [22-24]. Keywords for procedure as well as anatomical and territorial location were used. In addition, the references found in systematic reviews and meta-analyses were used to identify primary studies, a grey literature search was conducted, and key authors were contacted. 


\subsection{Inclusion and Exclusion Criteria and the Revision Process}

The PICO criteria (population, intervention, comparison, outcome) were used to identify studies. We included documents in English and Spanish that focused on the evaluation of effectiveness and safety in primary shoulder arthroplasties performed in public hospitals in Spain. Due to limitations related to the robustness of the data and possible biases when making inferences in the population, only studies with a sample size of 20 or more primary interventions were included. Documents that included patients under 18 years of age, studies that evaluated revision implants or those indicated for tumours or congenital diseases, and studies aimed at evaluating surgical techniques were excluded. Additionally, studies evaluating complications, adverse effects and/or effectiveness based on certain patient characteristics were excluded due to the difficulty in generalizing and comparing their results.

A screening of the title, summary and full text was carried out independently by two expert reviewers (JAT and XGC), while possible discrepancies were resolved by a third reviewer (KS). After study selection, a narrative synthesis of the evidence obtained was carried out. Given the variability in study characteristics and outcome variable presentation, a meta-analysis was deemed unfeasible. Therefore, the information was extracted in various tables of evidence with four dimensions: study design, arthroplasty characteristics, safety and effectiveness of primary shoulder arthroplasties. To evaluate the prosthesis models, the Orthopaedic Data Evaluation Panel (ODEP) scores were used [25]. ODEP is a panel of independent experts that publishes reference indexes to assess the effectiveness of different models of anatomical and reverse prostheses. The criteria used were based on implant survival, follow-up time and the size of the cohort analysed. Based on these criteria, implants were assigned to categories in terms of the evidence supporting their use.

\subsection{Quality of Studies Included}

The quality of the studies and their design were considered according to the Scottish Intercollegiate Guidelines Network (SIGN) levels of evidence hierarchy [26]. Furthermore, the Risk of Bias tool (RoB 2.0) was used for randomized trials to assess the quality of evidence [27], while for non-randomized trials, the Risk of Bias in Non-Randomized Studies of Interventions tool (ROBINS-I) was used [28]. Finally, for single-cohort study designs, the scale of evidence assessment for case series studies from the Institute of Health Economics was used [29].

SIGN levels were assigned according to the quality of the study evidence based on their design from the $1++$ level of higher evidence reserved for high-quality meta-analyses, systematic reviews of randomized clinical trials, and randomized clinical trials with very low risk of bias, to level 4 , which includes expert opinion. To compare studies, the risk of bias for each reference was calculated using the RoB 2.0 and ROBINS-I tools [27,28], assigning 4 points to "critical" risk assessments; 3 points to "high" risk; 2 points to "moderate" risk, "some considerations" or cases assessed with "insufficient information"; and 1 point for "low" risk assessment. Next, the percentage of points obtained over the total points possible for all categories was calculated. For single-cohort studies, the percentage of positive responses was obtained from the assessment scale.

\section{Results}

A total of 360 references were identified (259 from EMBASE, 84 from PubMed and 17 from SCIELO) (Figure 1). After eliminating duplicates, 323 were screened by title and abstract. After screening, 277 documents were excluded, thus including 46 for full-text review. Of these 46 references, 25 were excluded and 21 studies were included in the final evidence tables [18-20,30-47].

Table 1 shows the level of evidence of the selected studies. Two randomized clinical trials had a SIGN 1+ level and two non-randomized clinical trials a SIGN $2++$. The remaining studies were observational, 11 of which were retrospective. In terms of risk of bias assessment, little variability was observed in the risk attributed to the different studies. The greatest risk of bias came from subjectivity in measuring outcome variables, followed by the possible impact of uncontrolled confounding variables. 
The average range for patient follow-up was between 12 and 79 months, and included the results of 1293 arthroplasties. The number of cases per study ranged from 21 to 163 .

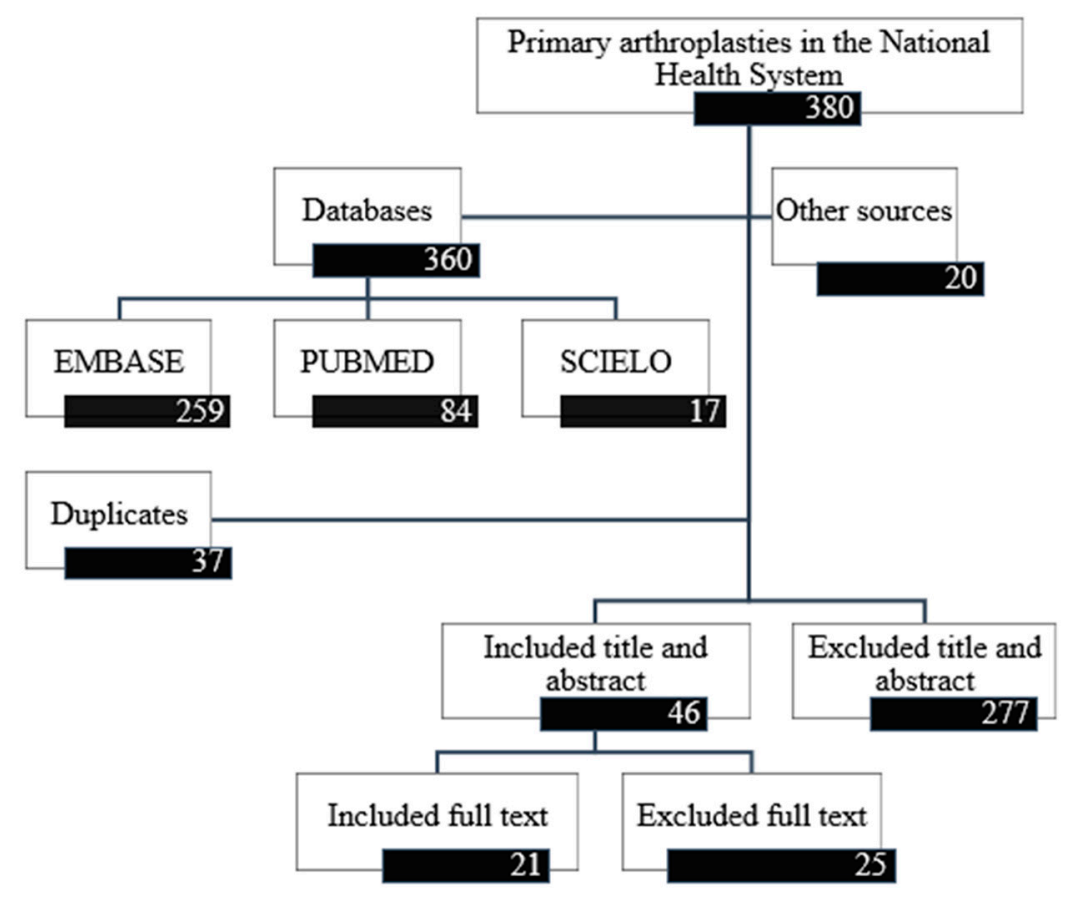

Figure 1. Study selection flow diagram.

Table 2 shows that the average age of patients included was over 70 years, approximately $70 \%$ of whom were women. In addition, two studies documented low comorbidity in their patients (Charlson Comorbidity Index less than 2) $[18,19]$. There were four main indications for undertaking primary arthroplasty: acute fractures and fracture-dislocations of the proximal humerus, rotator cuff arthropathy, fracture sequelae and malunion of the proximal humerus, and degenerative diseases. About half of the interventions were performed using a deltopectoral approach, while the rest used a superolateral or anterosuperior approach. In terms of implant characteristics and fixation, 18 studies included reverse prostheses, 8 of which used a cemented fixation and 6 of which were non-cemented. The most frequently used prosthesis models included in these studies were the Delta Xtend, with an ODEP assessment of 10A, and the Delta III, which is not evaluated by ODEP. The third most frequent was the Lima SMR, with an ODEP rating of 10A. 
Table 1. Primary shoulder arthroplasties. Evidence, risk of bias, and study design.

\begin{tabular}{|c|c|c|c|c|c|}
\hline Author, Year & $\begin{array}{l}\text { SIGN Level of } \\
\text { Evidence }\end{array}$ & Risk of Bias (\%) & Study Type (Time Period) & $\begin{array}{c}\text { Average Patient } \\
\text { Follow-Up in Months }\end{array}$ & $\begin{array}{c}\text { Total Number } \\
\text { of Cases }\end{array}$ \\
\hline Torrens et al., 2016 & $1+$ & 56 & Randomized controlled trial (2010-2012) & 24 & 81 \\
\hline Sebastià Forcada et al., 2014 & $1+$ & 56 & Randomized controlled trial (2009-2011) & 30 reverse and 28 partial & 61 \\
\hline Boyer et al., 2017 & $2++$ & 39 & Nonrandomized trial (2009-2011) & 15 reverse and 25 partial & 134 \\
\hline Alentorn-Geli et al., 2014 & $2++$ & 50 & Nonrandomized trial (2005-2012). & 40 reverse and 72 partial & 32 \\
\hline Jorge-Mora et al., 2018 & $2+$ & 43 & Retrospective observational (2012-2017) & 26 & 114 \\
\hline Sebastià Forcada et al. 2017 & $2+$ & 54 & Retrospective observational (2009-2011) & $\begin{array}{c}40 \text { plate failure and } 37 \\
\text { acute fracture }\end{array}$ & 60 \\
\hline Lopiz et al., 2016 & $2+$ & 54 & Retrospective observational (2009-2012) & 33 & 42 \\
\hline Bonilla et al., 2012 & $2-$ & 54 & Retrospective observational (2003-2011) & $\begin{array}{l}25 \text { Delta and } 9 \\
\text { Comprehensive }\end{array}$ & 43 \\
\hline Izquierdo-Fernández et al., 2017 & $2-$ & 46 & Prospective observational (2012) & 48 & 29 \\
\hline Cáceres-Sánchez et al., 2015 & 3 & 50 & Retrospective observational (2004-2012) & 36 & 52 \\
\hline Martinez et al., 2012 & 3 & 43 & Prospective observational (2003-2007) & 48 & 44 \\
\hline Zafra et al., 2014 & 3 & 43 & Prospective observational (2003-2011) & 51 & 35 \\
\hline García-Fernandez et al., 2015 & 3 & 39 & Retrospective observational (2003-2014) & 79 & 163 \\
\hline Alcobía-Díaz et al., 2017 & 3 & 43 & Retrospective observational (2009-2011) & 53 & 116 \\
\hline Torrens et al., 2016 & 3 & 43 & Prospective observational (NS) & 12 & 60 \\
\hline Torrens et al., 2017 & 3 & 36 & Prospective observational (NS) & 24 & 58 \\
\hline Torrens et al., 2018 & 3 & 36 & Retrospective observational (2010-2012) & 29 & 41 \\
\hline Delgado-Rodríguez et al., 2013 & 3 & 54 & Retrospective observational (2006-2010) & 17 & 40 \\
\hline Hernández-Elena et al., 2015 & 3 & 43 & Retrospective observational (2009-2013) & 18 & 37 \\
\hline Villodre-Jiménez et al., 2016 & 3 & 43 & Prospective observational (2008-2014) & 34 & 30 \\
\hline Andrés-Cano et al., 2014 & 3 & 43 & Retrospective observational (2009-2010) & 21 & 21 \\
\hline
\end{tabular}

SIGN: Scottish Intercollegiate Guidelines Network; NS: Not specified. 
Table 2. Characteristics of shoulder arthroplasties included in the selected studies.

\begin{tabular}{|c|c|c|c|c|c|}
\hline Author, Year & Diagnosis & Patient Characteristics & Characteristics of the Intervention & $\begin{array}{l}\text { Arthroplasty and } \\
\text { Fixation }\end{array}$ & ODEP Model Rating \\
\hline Torrens et al., 2016 & $\begin{array}{l}\text { Rotator cuff arthropathy }(82 \% \\
\text { G38 vs. } 63 \% \text { G } 42) \text {, Proximal } \\
\text { humeral fracture (18\% G38 vs. } \\
26 \% \text { G42) and fracture sequelae } \\
\text { (13\% G38 vs. } 11 \% \text { G42). }\end{array}$ & $\begin{array}{l}\text { Average age, } 75 \text { years G38 ( } 88 \% \\
\text { women) vs. } 76 \text { G42 ( } 84 \% \text { women). }\end{array}$ & $\begin{array}{l}\text { Glenoid component fixed without } \\
\text { retroversion, metaglene, flush. Deltopectoral } \\
\text { approach for sequelae fractures and anterior } \\
\text { superior in acute fractures, and pretension of } \\
\text { the rotator cuff. Movement starting at } 24 \mathrm{~h} \text {, } \\
\text { Sling: } 3 \text { weeks. }\end{array}$ & Reverse. & $\begin{array}{l}\text { Delta Xtend - } 10 \mathrm{~A} \text {; glenoid } \\
\text { component size } \\
(38 \text { vs. } 42 \mathrm{~mm}) .\end{array}$ \\
\hline $\begin{array}{l}\text { Sebastià Forcada et al., } \\
\qquad 2014\end{array}$ & $\begin{array}{l}\text { Proximal humeral fracture in } \\
\text { three (16\% reverse vs. } 13 \% \\
\text { partial) and four fragmented } \\
\text { ( } 84 \% \text { reverse vs. } 87 \% \text { partial). } \\
\text { Rotator cuff arthropathy: } 55 \% \\
\text { reverse vs. } 63 \% \text { partial. } \\
\end{array}$ & $\begin{array}{l}\text { Average age: } 75 \text { years, reverse } \\
\text { ( } 87 \% \text { women, } 61 \% \text { lesion in dominant } \\
\text { arm) vs. } 73 \text { years, partial } \\
\text { ( } 83 \% \text { women). }\end{array}$ & $\begin{array}{l}\text { Deltopectoral approach. Sling: } 3 \text { weeks. } \\
\text { Average time between fracture and surgery: } \\
5 \text { days. Rehabilitation: beginning of active } \\
\text { and passive movement at } 2 \text { weeks, active } \\
\text { with resistance at } 6 \text { weeks. }\end{array}$ & $\begin{array}{l}\text { Reverse vs. partial } \\
\text { (non-cemented). }\end{array}$ & Lima SMR - 10A. \\
\hline Boyer et al., 2017 & $\begin{array}{l}\text { Three- and four-part proximal } \\
\text { humeral fractures. }\end{array}$ & $\begin{array}{l}\text { Average age: } 78 \text { years, reverse vs. } \\
68 \text { years partial. }\end{array}$ & $\begin{array}{l}\text { Deltopectoral }(88 \%) \text { or superolateral }(12 \%) \\
\text { approach. Average time between fracture } \\
\text { and surgery: } 7 \text { days. }\end{array}$ & $\begin{array}{l}\text { Reverse vs. partial. } \\
\text { Non-cemented } \\
\text { (using two screws). }\end{array}$ & - \\
\hline Alentorn-Geli et al., 2014 & $\begin{array}{l}\text { Fracture sequelae of the proximal } \\
\text { humerus. }\end{array}$ & $\begin{array}{l}\text { Average age: } 79 \text { years, reverse } \\
\text { ( } 80 \% \text { women) vs. } 83 \text { years, partial } \\
\text { ( } 33 \% \text { women). }\end{array}$ & $\begin{array}{l}\text { Deltopectoral }(84 \%) \text { or superolateral }(16 \%) \\
\text { approach. Tuberosity osteotomy type IV. } \\
\text { Subscapularis repair in partial arthroplasties } \\
\text { and in the reverse deltopectoral approach. }\end{array}$ & Reverse vs. partial. & $\begin{array}{l}\text { Reverse - Delta Xtend - } \\
\text { 10A vs. Partial: Global } \\
\text { Advantage. }\end{array}$ \\
\hline Jorge-Mora et al., 2018 & Proximal humeral fracture. & $\begin{array}{l}\text { Average age: } 78 \text { years, cemented } \\
\text { ( } 100 \% \text { women) vs. } 76 \text { years, } \\
\text { non-cemented }(91 \% \text { women) } / 76 \text { years } \\
\text { union }(95 \% \text { women) vs. } 78 \text { years } \\
\text { non-union ( } 95 \% \text { women). }\end{array}$ & $\begin{array}{l}\text { Approach: deltopectoral cemented ( } 92 \% \text { ) vs. } \\
\text { non-cemented }(94 \%) / \text { deltopectoral union } \\
(92 \%) \text { vs. nonunion }(95 \%) \text {, the rest } \\
\text { superolateral. Average time between } \\
\text { fracture and surgery: } 9 \text { days cemented/8 } \\
\text { days union vs. } 8 \text { days nonunion. }\end{array}$ & $\begin{array}{l}\text { Total reverse: cemented } \\
\text { vs. non-cemented. }\end{array}$ & $\begin{array}{l}\text { Arrow shoulder fracture } \\
\text { and anatomic shoulder } \\
\text { reconstruction vs. } \\
\text { Humelock II. }\end{array}$ \\
\hline $\begin{array}{l}\text { Sebastià Forcada et al., } \\
\qquad 2017\end{array}$ & $\begin{array}{l}\text { Complex fracture sequela due to } \\
\text { fixation failure of proximal } \\
\text { humeral plate vs. proximal } \\
\text { humeral fracture. }\end{array}$ & $\begin{array}{l}\text { Average age: } 73 \text { years, sequela } \\
\text { (63\% women, } 43 \% \text { dominant arm) vs. } \\
75 \text { years, fracture ( } 63 \% \text { women). }\end{array}$ & $\begin{array}{l}\text { Deltopectoral approach. Average of } \\
2.311 \text { days between fracture and intervention } \\
\text { in the group with sequelae. Sling: } 3 \text { weeks. } \\
\text { Rehabilitation: started at } 3 \text { weeks, lasted } \\
4 \text { weeks. }\end{array}$ & $\begin{array}{l}\text { Total reverse, } \\
\text { non-cemented. }\end{array}$ & Lima SMR - 10A. \\
\hline Lopiz et al., 2016 & $\begin{array}{l}\text { Humerus fracture: three ( } 12 \% \text { vs. } \\
19 \% \text { ) and four ( } 42 \% \text { vs. } 48 \%) \\
\text { fragmented and dislocated } \\
\text { fractures }(46 \% \text { vs. } 37.5 \%) \text {. }\end{array}$ & $\begin{array}{l}\text { Over } 80 \text { years old vs. under } 80 \text { years } \\
\text { old. } 80 \% \text { women. Dominant } \\
\text { shoulder inured }(62 \%) \text {. }\end{array}$ & $\begin{array}{l}\text { Deltopectoral approach. Average of } 6 \text { days } \\
\text { between fracture and intervention. } \\
\text { Rehabilitation: passive movement from } 24 \mathrm{~h} \\
\text { post intervention to } 2 \text { weeks, exercises for } \\
\text { 3-4 weeks. }\end{array}$ & Reverse cemented. & Delta Xtend - 10A. \\
\hline Bonilla et al., 2012 & $\begin{array}{l}\text { Rotator cuff arthropathy or } \\
\text { osteoarthritis secondary to rotator } \\
\text { cuff tear. }\end{array}$ & $\begin{array}{l}\text { Average age: } 76 \text { years, Delta vs. } \\
(87.5 \% \text { women, } 81 \% \text { right arm) vs. } \\
72 \text { years Comprehensive } \\
(92 \% \text { women })\end{array}$ & $\begin{array}{l}\text { Approach: Delta Xtend transdeltoid vs. } \\
\text { Comprehensive deltopectoral. }\end{array}$ & Reverse. & $\begin{array}{l}\text { Delta Xtend - 10A vs. } \\
\text { Comprehensive Reverse } \\
\text { Shoulder System - 5A. }\end{array}$ \\
\hline
\end{tabular}


Table 2. Cont.

\begin{tabular}{|c|c|c|c|c|c|}
\hline Author, Year & Diagnosis & Patient Characteristics & Characteristics of the Intervention & $\begin{array}{l}\text { Arthroplasty and } \\
\text { Fixation }\end{array}$ & ODEP Model Rating \\
\hline $\begin{array}{l}\text { Izquierdo-Fernández } \\
\text { et al., } 2017\end{array}$ & $\begin{array}{l}\text { Rotator cuff arthropathy, } \\
\text { fractures, or dislocations. }\end{array}$ & $\begin{array}{l}\text { Average age: } 78 \text { years ( } 80 \% \text { women). } \\
\text { Body mass index } \leq 35 \text { vs. }>35 \text {. }\end{array}$ & - & Reverse. & Delta Xtend - 10A. \\
\hline $\begin{array}{l}\text { Cáceres-Sánchez et al., } \\
2015\end{array}$ & $\begin{array}{l}\text { Proximal humeral fracture }(10 \%) \text {, } \\
\text { prosthetic revision }(12 \%) \text {, fracture } \\
\text { sequelae }(19 \%) \text {, rotator cuff tear } \\
(60 \%) .\end{array}$ & Average age: 70 years ( $84 \%$ women). & $\begin{array}{l}\text { Deltopectoral approach. Sling: } 3 \text { weeks. } \\
\text { Rehabilitation: passive movements between } \\
\text { the 1st and 3rd weeks, active movements } \\
\text { between the } 2 \text { nd and } 4 \text { th weeks and muscle } \\
\text { enhancement between the 3rd and } \\
\text { 12th weeks. }\end{array}$ & Reverse. & $\begin{array}{l}\text { Delta Xtend - 10A (58\%) } \\
\text { and Aequalis Reversed - } \\
\text { 5A (42\%). }\end{array}$ \\
\hline Martinez et al., 2012 & $\begin{array}{l}\text { Fracture sequelae of the proximal } \\
\text { humerus. }\end{array}$ & Average age: 77 years ( $60 \%$ women). & $\begin{array}{l}\text { Deltopectoral approach: Average time } \\
\text { between fracture and surgery: } 365 \text { days. } \\
\text { Rehabilitation: starting at } 3 \text { weeks. }\end{array}$ & $\begin{array}{l}\text { Reverse cemented ( } 45 \%) \\
\text { or non-cemented }(55 \%) \text {. }\end{array}$ & Lima SMR - 10A. \\
\hline Zafra et al., 2014 & $\begin{array}{l}\text { Error in the treatment of proximal } \\
\text { humeral fractures in two }(40 \%) \text {, } \\
\text { three }(26 \%) \text {, and four parts }(34 \%) \text {. }\end{array}$ & $\begin{array}{l}\text { Average age: } 69 \text { years. Fracture in } \\
\text { dominant arm }(86 \%) \text {. }\end{array}$ & Deltopectoral approach. & Reverse cemented. & Delta III. \\
\hline $\begin{array}{l}\text { García-Fernandez et al., } \\
2015\end{array}$ & $\begin{array}{l}\text { Rotator cuff injury }(30 \%) \text {, rotator } \\
\text { cuff arthropathy }(44 \%) \text {, proximal } \\
\text { humeral fractures }(26 \%) \text {. }\end{array}$ & $\begin{array}{l}\text { Average age: } 76 \text { years ( } 87.5 \% \text { women) } \\
\text { for the sample that included } \\
40 \text { additional revision arthroplasties. }\end{array}$ & $\begin{array}{l}\text { Approach: deltopectoral (fractures) or } \\
\text { superolateral (rotator cuff tears and } \\
\text { arthropathies) }\end{array}$ & $\begin{array}{l}\text { Reverse (cemented and } \\
\text { non-cemented). }\end{array}$ & $\begin{array}{c}\text { Delta III (9\%), Delta Xtend } \\
(43 \%)-10 \mathrm{~A}, \text { Lima SMR } \\
(29 \%) .\end{array}$ \\
\hline Alcobía-Díaz et al., 2017 & Rotator cuff arthropathy. & $\begin{array}{l}\text { Average age: } 81 \text { years ( } 88 \% \text { women) } \\
\text { Charlson Comorbidity Index: } \\
1.7 \text { = low comorbidity. }\end{array}$ & $\begin{array}{l}\text { Superolateral approach }(76 \%) \text { or } \\
\text { deltopectoral }(24 \%) \text {. Passive rehabilitation } \\
\text { during hospital stay, exercises for } 6 \text { weeks. }\end{array}$ & Total. & - \\
\hline Torrens et al., 2016 & Rotator cuff arthropathy. & $\begin{array}{l}\text { Average age: } 74.5 \text { years } \\
\text { ( } 92 \% \text { women). }\end{array}$ & & Reverse. & Delta Xtend - 10A. \\
\hline Torrens et al., 2017 & $\begin{array}{l}\text { Reverse }(71 \%): 43 \% \text { rotator cuff } \\
\text { arthropathy, } 28 \% \text { proximal } \\
\text { humeral fractures. Total: } 22 \% \\
\text { primary osteoarthritis. Partial: } \\
7 \% \text { proximal humeral fractures. }\end{array}$ & $\begin{array}{l}\text { Average age: fractures, } 74 \text { years; } \\
\text { osteoarthritis, } 78 \text { years; arthropathy, } \\
74 \text { years ( } 88 \% \text { women). }\end{array}$ & $\begin{array}{l}\text { Reverse: anterosuperior approach. } \\
\text { Anatomic: deltopectoral approach. Average } \\
\text { time between fracture and surgery: } 11 \text { days. }\end{array}$ & $\begin{array}{c}\text { Total anatomic }(23 \%), \\
\text { partial }(7 \%) \text { and reverse } \\
(70 \%) .\end{array}$ & $\begin{array}{l}\text { Reverse: Delta Xtend - 7A, } \\
\text { Total: Global AP - 5A, } \\
\text { Partial: Global Unite. }\end{array}$ \\
\hline Torrens et al., 2018 & $\begin{array}{l}\text { Proximal humeral fracture in } \\
\text { three }(17 \%) \text { or four parts }(83 \%) \text {. }\end{array}$ & $\begin{array}{l}\text { Average age: } 78 \text { years } \\
\text { ( } 87.5 \% \text { women). Average BMI: } 28 .\end{array}$ & $\begin{array}{l}\text { Anterosuperior approach. Average time } \\
\text { between fracture and surgery: } 12 \text { days. }\end{array}$ & Reverse cemented. & Delta Xtend - 10A. \\
\hline $\begin{array}{l}\text { Delgado-Rodríguez et al., } \\
2013\end{array}$ & Proximal humeral fracture. & $\begin{array}{l}\text { Average age: } 76 \text { years } \\
\text { (87.5\% women). }\end{array}$ & $\begin{array}{l}\text { Rehabilitation: average start at } 4 \text { weeks. } \\
\text { Average number of sessions }=39\end{array}$ & Partial. & \\
\hline $\begin{array}{l}\text { Hernández-Elena et al., } \\
\qquad 2015\end{array}$ & $\begin{array}{l}\text { Proximal humeral fracture with } \\
\text { risk of osteonecrosis of the } \\
\text { humeral head. Type of fracture: } \\
\text { four fragments ( } 54 \%) \text {, three } \\
\text { fragments ( } 30 \%) \text {, } \\
\text { fracture-dislocation }(16 \%) \text {. }\end{array}$ & Average age: 77 years ( $97 \%$ women). & $\begin{array}{l}\text { Deltopectoral approach. Rehabilitation: } \\
\text { starting at } 2 \text { weeks with passive movement, } \\
\text { resistance exercises starting at } 6 \text { weeks. }\end{array}$ & Reverse. & $\begin{array}{c}\text { Aequalis }^{\circledR} \text { Reversed II - } \\
\text { 5A. }\end{array}$ \\
\hline
\end{tabular}


Table 2. Cont.

\begin{tabular}{|c|c|c|c|c|c|}
\hline Author, Year & Diagnosis & Patient Characteristics & Characteristics of the Intervention & $\begin{array}{l}\text { Arthroplasty and } \\
\text { Fixation }\end{array}$ & ODEP Model Rating \\
\hline $\begin{array}{l}\text { Villodre-Jiménez et al., } \\
2016\end{array}$ & $\begin{array}{l}\text { Humerus fracture in three parts } \\
(27 \%) \text { and four parts }(73 \%) \text {. With } \\
\text { non-reconstructible fractures, risk } \\
\text { of avascular necrosis, severe } \\
\text { osteoporosis and previous rotator } \\
\text { cuff injuries. }\end{array}$ & Average age: 75 years ( $87 \%$ women). & $\begin{array}{l}\text { Deltopectoral approach. Sling: } 3 \text { weeks. } \\
\text { Rehabilitation: passive movement starting at } \\
3 \text { weeks, exercises starting at } 6 \text { weeks. }\end{array}$ & Reverse cemented. & Lima SMR - 10A. \\
\hline Andrés-Cano et al., 2014 & $\begin{array}{l}\text { Proximal humeral fracture in } \\
\text { three }(10 \%) \text { or four parts }(57 \%) \text {, } \\
\text { fracture-dislocation }(33 \%) .\end{array}$ & $\begin{array}{l}\text { Average age: } 72 \text { years ( } 90 \% \text { women). } \\
\text { Charlson Comorbidity: } 0 \text { or } 1 .\end{array}$ & $\begin{array}{l}\text { Deltopectoral approach. Average time } \\
\text { between fracture and surgery: } 17 \text { days. Sling: } \\
5 \text { weeks. Rehabilitation starting at } 4 \text { weeks. }\end{array}$ & Partial non-cemented. & $\begin{array}{l}\text { Epoca Shoulder } \\
\text { Arthroplasty System. }\end{array}$ \\
\hline
\end{tabular}

ODEP: Orthopaedic Data Evaluation Panel; G38: 38 mm glenosphere; G42: 42 mm glenosphere; \%: percentage; BMI: body mass index; -: no information available. 
Table 3 shows that the most frequent complication in reverse shoulder arthroplasties was scapular notching, reported in 14 studies [33,48]. One study found a higher presence in older patients [46]. In terms of complications associated with tuberosities, malunion was documented in six studies, with the maximum rate being $33 \%$. Additionally, resorption of the tuberosities was reported in five studies. Prosthesis infection was documented in seven of the included studies, with one study citing up to $8 \%$ [34]. Intraoperative fractures were also documented in seven studies, with the highest values being $7 \%$. Similarly, periprosthetic fracture in five documents and ossification in one were also seen. There was a $1 \%$ dislocations rate, and less than $1 \%$ were complications relating to fixation, positioning, and movement of the prosthesis. The main neurological vascular and lymphatic system complications were paralysis in three studies and hematoma in three others. In terms of prosthesis survival, $67 \%$ of the studies selected cited approximately $6 \%$ revision rate between 12 and 78 months. A significant difference was found in the revision rate in two studies comparing partial arthroplasties (hemiarthroplasty) to reverse arthroplasties [32,34]. The revision rate for hemiarthroplasties was far higher, with a difference greater than $15 \%$ in both studies.

In terms of the effectiveness of shoulder arthroplasties, Table 4 shows that in the studies evaluating patients before the intervention, the Constant-Murley test score was approximately $30 \%$. After the intervention, the average score was approximately $65 \%$, with significant improvement reported in four studies $[20,30,34,41]$. Seven of the eight studies included metrics before and after reported improvement in external rotation, with three studies being statistically significant. In one study, better results in terms of hemiarthroplasties were observed in reverse prostheses [32]. Five of the seven studies that dealt with internal rotation and analysed metrics before and after reported improvements in movement. One of these studies had a significant difference. Better results were observed in patients that had fractures with tuberosity union compared to a group of patients with tuberosity malunion [35]. Sixteen of the 21 studies considered other results, highlighting their frequency on the UCLA Shoulder Rating Scale (UCLA) in two studies, Disabilities of the Arm, Shoulder and Hand (DASH) or QuickDASH in six studies and on Visual Analog Scale (VAS) in four studies. 
Table 3. Reliability of primary shoulder arthroplasties.

\begin{tabular}{|c|c|c|c|c|c|c|c|}
\hline Author, Year & Scapular Notching & Infection & $\begin{array}{l}\text { Fractures, Tears and } \\
\text { Ossifications }\end{array}$ & $\begin{array}{l}\text { Complications in } \\
\text { Tuberosities }\end{array}$ & $\begin{array}{c}\text { Fixation, Dislocation or } \\
\text { Stiffness } \\
\text { Complications }\end{array}$ & $\begin{array}{l}\text { Neurological, Vascular } \\
\text { and/or Lymphatic } \\
\text { Complications }\end{array}$ & $\begin{array}{c}\text { Reinterventions and } \\
\text { Revisions }\end{array}$ \\
\hline Torrens et al., 2016 & $\begin{array}{c}\text { Scapular neck notching } \\
\text { (with intention to treat) } \\
46 \% \text { G38 vs. } 30 \% \text { G42. }\end{array}$ & $\begin{array}{c}0 \% \text { G38 vs. } 3 \% \\
\text { G42 }\end{array}$ & - & - & $\begin{array}{l}\text { Dislocation: } 3 \% \mathrm{G} 38 \text { vs. } \\
2 \% \mathrm{G} 42 .\end{array}$ & - & $\begin{array}{c}\text { Revision: } 3 \% \text { G38 vs. } \\
\text { 2\% G42. }\end{array}$ \\
\hline $\begin{array}{l}\text { Sebastià Forcada et al., } \\
\qquad 2014\end{array}$ & $\begin{array}{c}\text { Scapular neck notching: } \\
\text { 3\% Reverse vs. } 0 \% \\
\text { partial. }\end{array}$ & $\begin{array}{l}3 \% \text { reverse vs. } 3 \% \\
\text { partial. }\end{array}$ & $\begin{array}{c}\text { Intraoperative fracture: } \\
0 \% \text { reverse vs. } 3 \% \\
\text { partial. Ossification: } \\
16 \% \text { reverse vs. } 20 \% \\
\text { partial }\end{array}$ & $\begin{array}{l}\text { Malunion: } 19 \% \text { reverse } \\
\text { vs. } 13 \% \text { partial }(p=0.4) \text {. } \\
\text { Resorption: } 16 \% \text { reverse } \\
\text { vs. } 30 \% \text { partial }(p=0.4) .\end{array}$ & $\begin{array}{c}\text { Rigidity: } 0 \% \text { reverse vs. } \\
\text { 3\% partial. Migration: } \\
0 \% \text { reverse vs. } 20 \% \\
\text { partial. Radiolucency: } \\
13 \% \text { reverse vs. } 10 \% \\
\text { partial. }\end{array}$ & $\begin{array}{l}\text { Hematoma: } 0 \% \text { reverse } \\
\text { vs. } 4 \% \text { partial. }\end{array}$ & $\begin{array}{l}\text { Revision before the } \\
\text { 40th month: } 3 \% \\
\text { reverse vs. } 20 \% \\
\text { partial. }\end{array}$ \\
\hline Boyer et al., 2017 & $\begin{array}{l}\text { Notching: Reverse ( } 8 \%) \\
\text { vs. partial }(0 \%) .\end{array}$ & - & $\begin{array}{c}\text { Periprosthetic fracture: } \\
3 \% \text { reverse vs. } 1 \% \\
\text { partial. Cuff tear: } 0 \% \\
\text { reverse vs. } 5 \% \text { partial. }\end{array}$ & $\begin{array}{c}\text { Lysis } 6 \% \text { reverse vs. } 2 \% \\
\text { partial. }\end{array}$ & $\begin{array}{l}\text { Poor fixation: } 7 \% \\
\text { reverse vs. } 2 \% \text { partial. }\end{array}$ & $\begin{array}{c}\text { Phlebitis: } 3 \% \text { reverse vs. } \\
0 \% \text { partial. Paralysis: } 1 \% \\
\text { reverse vs. } 1 \% \text { partial. } \\
\text { Lymphedema: } 0 \% \\
\text { reverse vs. } 2 \% \text { partial. }\end{array}$ & $\begin{array}{l}\text { Revision: } 7 \% \text { reverse } \\
\text { vs. } 2 \% \text { partial. }\end{array}$ \\
\hline $\begin{array}{l}\text { Alentorn-Geli et al., } \\
2014\end{array}$ & $\begin{array}{l}\text { Glenoid erosion: } 0 \% \\
\text { reverse vs. } 8 \% \text { partial. }\end{array}$ & $\begin{array}{l}0 \% \text { reverse vs. } 8 \% \\
\text { partial. }\end{array}$ & - & - & - & - & $\begin{array}{l}\text { Revision: } 0 \% \text { reverse } \\
\text { vs. } 25 \% \text { partial. }\end{array}$ \\
\hline Jorge-Mora et al., 2018 & - & $\begin{array}{l}\text { Early infection: } 0 \% \\
\text { cemented vs. } 3 \% \\
\text { non-cemented. }\end{array}$ & $\begin{array}{l}\text { Periprosthetic fracture: } \\
0 \% \text { cemented vs. } 3 \% \\
\text { non-cemented. }\end{array}$ & $\begin{array}{c}\text { Poor reinforcement: } \\
33 \%, 54 \% \text { cemented vs. } \\
76 \% \text { non-cemented ( } p= \\
0.07 \text { ). }\end{array}$ & $\begin{array}{c}\text { Dislocation: } 0 \% \\
\text { cemented vs. } 3 \% \\
\text { non-cemented. }\end{array}$ & $\begin{array}{l}\text { Paralysis: } 0 \% \text { cemented } \\
\text { vs. } 3 \% \text { non-cemented. }\end{array}$ & $\begin{array}{l}\text { Revision: } 0 \% \\
\text { cemented vs. } 9 \% \\
\text { non-cemented. }\end{array}$ \\
\hline $\begin{array}{l}\text { Sebastià Forcada et al., } \\
\qquad 2017\end{array}$ & $0 \%$ & - & $\begin{array}{c}\text { Acromion fracture: } 3 \% \\
\text { sequela vs. } 0 \% \text { acute. } \\
\text { Intraoperative fracture: } \\
0 \% \text { sequelae vs. } 3 \% \\
\text { acute. }\end{array}$ & - & $\begin{array}{c}\text { Dislocation: } 7 \% \text { sequela } \\
\text { vs. } 0 \% \text { acute. Loosening } \\
3 \% \text { sequela vs. } 0 \% \text { acute. } \\
\text { Radiolucency: } 7 \% \\
\text { sequela vs. } 0 \% \text { fracture. }\end{array}$ & - & $\begin{array}{l}\text { Revision: } 13 \% \text { sequela } \\
\text { vs. } \% \text { fracture. }\end{array}$ \\
\hline Lopiz et al., 2016 & Scapular notching: $14 \%$. & & $\begin{array}{l}\text { Periprosthetic fracture: } \\
2 \% .\end{array}$ & $\begin{array}{l}\text { Malunion: } 19 \% . \\
\text { Resorption: } 5 \% .\end{array}$ & $\begin{array}{c}\text { Dislocation: } 2 \% . \\
\text { Radiolucency: } 0 \% .\end{array}$ & Hematoma: $4 \%$. & Revision: $2 \%$. \\
\hline Bonilla et al., 2012 & $\begin{array}{c}\text { Scapular notching: } 31 \% \\
\text { Delta vs. } 9 \% . \\
\text { Comprehensive. }\end{array}$ & $\begin{array}{l}6 \% \text { Delta vs. } 0 \% \\
\text { Comprehensive }\end{array}$ & - & - & $\begin{array}{l}\text { Migration: } 3 \% \text { Delta vs. } \\
0 \% \text { Comprehensive. }\end{array}$ & - & $\begin{array}{l}\text { Revision: } 9 \% \text { Delta vs. } \\
0 \% \text { Comprehensive. }\end{array}$ \\
\hline $\begin{array}{l}\text { Izquierdo-Fernández } \\
\text { et al., } 2017\end{array}$ & $\begin{array}{c}\text { Scapular notching: } 47 \% \\
\text { BMI < 35 vs. } 50 \% \\
\text { BMI }>35 .\end{array}$ & - & - & - & $\begin{array}{c}\text { Radiolucency: } 57 \% \text { vs. } \\
\quad 37.5 \%(p=0.3)\end{array}$ & - & - \\
\hline $\begin{array}{l}\text { Cáceres-Sánchez et al., } \\
2015\end{array}$ & Scapular notching: $17 \%$. & $4 \%$ & $\begin{array}{c}\text { Acromion fracture: } 2 \% . \\
\text { Intraoperative fracture: } \\
2 \% .\end{array}$ & - & $\begin{array}{l}\text { Radiolucency: } 2 \% \\
\text { Instability: } 4 \% \\
\text { Loosening: } 4 \% .\end{array}$ & - & Reintervention: $9 \%$. \\
\hline
\end{tabular}


Table 3. Cont.

\begin{tabular}{|c|c|c|c|c|c|c|c|}
\hline Author, Year & Scapular Notching & Infection & $\begin{array}{l}\text { Fractures, Tears and } \\
\text { Ossifications }\end{array}$ & $\begin{array}{l}\text { Complications in } \\
\text { Tuberosities }\end{array}$ & $\begin{array}{c}\text { Fixation, Dislocation or } \\
\text { Stiffness } \\
\text { Complications }\end{array}$ & $\begin{array}{l}\text { Neurological, Vascular } \\
\text { and/or Lymphatic } \\
\text { Complications }\end{array}$ & $\begin{array}{l}\text { Reinterventions and } \\
\text { Revisions }\end{array}$ \\
\hline Martinez et al., 2012 & $\begin{array}{l}\text { Glenoid notching: } 41 \% \text {. } \\
\text { Scapular notching: } 41 \% \text {. }\end{array}$ & - & - & Resorption: $7 \%$. & $\begin{array}{l}\text { Radiolucency: } 11 \% . \\
\text { Dislocation: } 14 \% . \\
\text { Loosening: } 2 \% .\end{array}$ & Paralysis $1 \%$. & Revision: $11 \%$. \\
\hline Zafra et al., 2014 & $60 \%$ & - & $\begin{array}{l}\text { Intraoperative fracture: } \\
5 \% \text {. Periprosthetic } \\
\text { fracture: } 2 \% .\end{array}$ & - & $\begin{array}{c}\text { Radiolucency: } 65 \% \\
\text { humeral, } 31.5 \% \text { glenoid. }\end{array}$ & - & $\begin{array}{c}\text { Other complications } \\
(20 \%) .\end{array}$ \\
\hline $\begin{array}{l}\text { García-Fernandez } \\
\text { et al., } 2015\end{array}$ & - & - & $\begin{array}{l}\text { Periprosthetic humerus } \\
\text { fracture: intraoperative } \\
\text { with non-cemented } \\
\text { Lima SMR }(1 \%), \\
\text { postoperative with Lima } \\
\text { SMR }(1 \%) .\end{array}$ & - & - & - & - \\
\hline $\begin{array}{l}\text { Alcobía-Díaz et al., } \\
2017\end{array}$ & - & - & - & - & - & - & $\begin{array}{c}10 \% \text { analgesic } \\
\text { treatment } 6 \text { weeks } \\
\text { after the intervention }\end{array}$ \\
\hline Torrens et al., 2016 & - & - & - & - & - & - & - \\
\hline Torrens et al., 2017 & - & $0 \%$. & - & - & - & - & - \\
\hline Torrens et al., 2018 & Scapular notching: $15 \%$. & - & $\begin{array}{c}\text { Presence of osteophyte: } \\
12 \% .\end{array}$ & $\begin{array}{l}\text { Malunion: } 32 \% . \\
\text { Resorption: } 10 \% \text {. Poorer } \\
\text { union, depending on } \\
\text { comorbidity. }\end{array}$ & - & Paraesthesia: $15 \%$. & Revision: $2 \%$. \\
\hline $\begin{array}{l}\text { Delgado-Rodríguez } \\
\text { et al., } 2013\end{array}$ & - & - & - & - & - & - & - \\
\hline $\begin{array}{l}\text { Hernández-Elena } \\
\text { et al., } 2015\end{array}$ & $\begin{array}{l}\text { Scapular notching }(29 \%) \text {. } \\
\text { Relationship between } \\
\text { notching and age. }\end{array}$ & - & $\begin{array}{l}\text { Intraoperative fracture: } \\
\qquad 2 \% .\end{array}$ & - & - & $\begin{array}{l}\text { Hematoma: } 5 \% \text {. } \\
\text { Neuropraxia: } 3 \% \text {. }\end{array}$ & - \\
\hline $\begin{array}{l}\text { Villodre-Jiménez et al., } \\
2016\end{array}$ & $\begin{array}{c}\text { Scapular notching } \\
(46 \%) .\end{array}$ & - & $\begin{array}{l}\text { Intraoperative fracture: } \\
7 \% .\end{array}$ & Malunion: 33\%. & - & - & $\begin{array}{l}\text { Other complications } \\
46 \% \text {. }\end{array}$ \\
\hline $\begin{array}{l}\text { Andrés-Cano et al., } \\
2014\end{array}$ & - & - & - & $\begin{array}{l}\text { Malunion: } 5 \% . \\
\text { Resorption: } 24 \% \text {. }\end{array}$ & Radiolucency: 5\%. & - & - \\
\hline
\end{tabular}

G38: 38 mm glenosphere; G42: 42 mm glenosphere; BMI: Body Mass Index; $p$ : $p$-value; -: no information available. 
Table 4. Effectiveness of primary shoulder arthroplasties.

\begin{tabular}{|c|c|c|c|}
\hline Author, Year & Constant-Murley Score & Joint Assessment, by Constant-Murley Score & Other Results \\
\hline Torrens et al., 2016 & $\begin{array}{l}\text { Global score: } 29 \text { before }-57 \text { after G } 38 \text { vs. } \\
26 \text { before- } 55 \text { after G } 42 \text {. Pain: } 5 \\
\text { before-11 after G38 vs. } 5 \text { before- } 11 \\
\text { after G42. Daily activities: } 8 \text { before- }-14 \\
\text { after G38 vs. } 7 \text { before }-14 \text { after G42. }\end{array}$ & $\begin{array}{l}\text { Flexion }=4 \text { before vs. } 7 \text { after G38 vs. } 3 \text { before }-7 \text { after G42. } \\
\text { Abduction }=4 \text { before vs. } 6 \text { after G38 vs. } 3 \text { before- }-6 \text { after } \\
\text { G42. External rotation }=2 \text { before }-5 \text { after G38 vs. } 2 \\
\text { before- } 4 \text { after G42 (among groups after, } p=0.06 \text { ). Internal } \\
\text { rotation }=4 \text { before G38- } 5 \text { after vs. } 4 \text { before }-7 \text { after G42. } \\
\text { Strength }=2 \text { before }-8 \text { after G38 vs. } 2 \text { before- }-7 \text { after G42. }\end{array}$ & - \\
\hline Sebastià Forcada et al., 2014 & $\begin{array}{l}\text { Global score: } 80 \text { reverse vs. } 56 \text { partial. } \\
\text { Pain: } 14 \text { reverse vs. } 9 \text { partial. Activity: } \\
17 \text { reverse vs. } 12 \text { partial*. }\end{array}$ & $\begin{array}{c}\text { Flexion }=120^{\circ} \text { reverse } 80^{\circ} \text { partial }{ }^{*} \text {. Abduction }=113^{\circ} \\
\text { reverse vs. } 79^{\circ} \text { partial. External rotation }=5 \text { reverse vs. } \\
3 \text { partial. Internal rotation }=3 \text { reverse vs. } 3 \text { partial }(p=0.9) .\end{array}$ & $\begin{array}{l}\text { UCLA score: } 29 \text { reverse vs. } 21 \text { partial. } \\
\text { DASH score: } 17 \text { reverse vs. } 24 \text { partial. }\end{array}$ \\
\hline Boyer et al., 2017 & $\begin{array}{l}\text { Standard global score: } 72 \text { reverse vs. } \\
72 \text { partial. }\end{array}$ & $\begin{array}{l}\text { Flexion }=109^{\circ} \text { reverse vs. } 99.5^{\circ} \text { partial. Abduction }=99^{\circ} \\
\text { reverse vs. } 90^{\circ} \text { partial. External rotation }=21^{\circ} \text { reverse vs. } \\
28^{\circ} \text { partial. }\end{array}$ & QuickDASH: 36 reverse vs. 78 partial. \\
\hline Alentorn-Geli et al., 2014 & $\begin{array}{l}\text { Standard global score: } 35 \text { before vs. } \\
57 \text { after, no improvement in internal } \\
\text { rotation. Greater difference before vs. } \\
\text { after in reverse arthroplasties (standard } \\
\text { global, front flexion and activity level). }\end{array}$ & - & Score SF-36 (quality of life): No difference. \\
\hline Jorge-Mora et al., 2018 & $\begin{array}{l}\text { Global score: } 53 \text { cemented vs. } \\
60 \text { non-cemented/ } 63 \text { union vs. } 45 \\
\text { non-union (difference of } 15 \text { points } \\
\text { improvement). }\end{array}$ & $\begin{array}{l}\text { Abduction }=92^{\circ} \text { cemented vs. } 104^{\circ} \text { non-cemented } / 115^{\circ} \\
\text { union vs. } 68^{\circ} \text { non-union. Flexion }=92^{\circ} \text { cemented vs. } 106^{\circ} \\
\text { non-cemented } / 115^{\circ} \text { union vs. } 69^{\circ} \text { non-union. Internal } \\
\text { rotation }=35^{\circ} \text { cemented vs. } 36^{\circ} \text { non-cemented } 38^{\circ} \text { union vs. } \\
31^{\circ} \text { non-union. External rotation }=17^{\circ} \text { cemented vs. } 23^{\circ} \\
\text { non-cemented } / 28^{\circ} \text { union vs. } 5^{\circ} \text { non-union. }\end{array}$ & - \\
\hline Sebastià Forcada et al., 2017 & $\begin{array}{l}\text { Last standard global assessment: } \\
67 \text { sequela vs. } 78 \text { fracture. }\end{array}$ & $\begin{array}{l}\text { Strength }=2 \text { sequela vs. } 4 \text { fracture. Flexion }=114^{\circ} \text { sequela } \\
\text { vs. } 127^{\circ} \text { fracture. Abduction }=104^{\circ} \text { sequela vs. } 120^{\circ} \\
\text { fracture. External rotation }=4 \text { sequela vs. } 5 \text { fracture } \\
(p=0.3) \text {. Internal rotation }=3 \text { sequela vs. } 3 \text { fracture } \\
\qquad(p=0.7) .\end{array}$ & $\begin{array}{l}\text { Last assessment: Pain VAS } 8 \text { sequela vs. } 8 \text { fracture }(p=0.9) \text {, } \\
\text { UCLA score: } 26 \text { sequela vs. fracture } 29 \text { fracture, QuickDASH } \\
\text { score: } 21.5 \text { sequela vs. } 25 \text { fracture. Satisfied patients: } 93 \% \text {. }\end{array}$ \\
\hline Lopiz et al., 2016 & Global score: Lower in the older group. & $\begin{array}{l}\text { No differences among groups at } 24 \text { months post } \\
\text { intervention. }\end{array}$ & $\begin{array}{c}\text { DASH score: } 27 \text { older group vs. } 31 \text { younger group ( } p=0.1) \text {. } \\
\text { Problems reported in EQ-5D: anxiety ( } 38 \% \text { older vs. } 12 \% \\
\text { younger), pain/discomfort ( } 23 \% \text { vs. } 12 \%) \text {, activity ( } 38 \% \text { vs. } \\
0 \% \text {, self-care ( } 46 \% \text { vs. } \% \text { ) and mobility ( } 46 \% \text { vs. } 12 \%) \text {. } \\
\text { Health status and quality of life EQ-VAS: } 63 \text { younger vs. } \\
74 \text { older. }\end{array}$ \\
\hline Bonilla et al., 2012 & $\begin{array}{l}\text { Global score: } 32 \text { before }-57.5 \text { after Delta } \\
\text { vs. } 31 \text { before }-60 \text { after Comprehensive. }\end{array}$ & $\begin{array}{c}\text { Flexion (before-after) }=95^{\circ}-130^{\circ} \text { Delta vs. } 102^{\circ}-132^{\circ} \\
\text { Comprehensive. Abduction (before-after) }=86^{\circ}-123^{\circ} \\
\text { Delta vs. } 98^{\circ}-118^{\circ} \text { Comprehensive. Internal rotation } \\
\text { (before-after) }=46 \%-44 \% \text { Delta vs. } 49 \%-61 \% \\
\text { Comprehensive. External rotation (before-after) = } \\
43 \%-64 \% \text { Delta vs. } 51 \%-61 \% \text { Comprehensive. }\end{array}$ & - \\
\hline Izquierdo-Fernández et al., 2017 & - & - & ASES score: 75 vs. 63 . Length of stay: 5 vs. 6 days $(p=0.3)$. \\
\hline
\end{tabular}


Table 4. Cont

\begin{tabular}{|c|c|c|c|}
\hline Author, Year & Constant-Murley Score & Joint Assessment, by Constant-Murley Score & Other Results \\
\hline Cáceres-Sánchez et al., 2015 & Global score: 23 before -67 at 12 months. & $\begin{array}{c}\begin{aligned} \text { External rotation } & =26^{\circ} \text { before }-67^{\circ} \text { after. Flexion }=74^{\circ} \\
& \text { before }-135^{\circ} \text { after }\end{aligned}\end{array}$ & $\begin{array}{c}\text { There are no metrics for fractures before the intervention. } \\
\text { EQ-VAS: } 8 \text { before vs. } 2 \text { at one-year follow-up. Satisfied } \\
\text { patients: } 100 \% \text {. }\end{array}$ \\
\hline Martinez et al., 2012 & Global score: 28 before -58 after. & $\begin{array}{l}\text { Flexion }=40^{\circ} \text { before vs. } 100^{\circ} \text { after. Abduction }=41^{\circ} \text { before } \\
\text { vs. } 95^{\circ} \text { after. External rotation }=15^{\circ} \text { before vs. } 35 \text { after. } \\
\text { Internal rotation }=25^{\circ} \text { before vs. } 60^{\circ} \text { after. }\end{array}$ & $\begin{array}{l}\text { Estimation of proximity to a normal back: } 13 \% \text { before to } 56 \% \\
\text { after. Patient satisfaction: } 86 \% \text { satisfied or very satisfied. }\end{array}$ \\
\hline Zafra et al., 2014 & Global score: 23 before- -65.5 after. & $\begin{array}{c}\text { Flexion }=45^{\circ} \text { before vs. } 117^{\circ} \text { after. Abduction }=39^{\circ} \text { before } \\
\text { vs. } 96^{\circ} \text { after*. Internal rotation (no difference). External } \\
\text { rotation }=5^{\circ} \text { before vs. } 15.5^{\circ} \text { after. }\end{array}$ & $\begin{array}{l}\text { Cofield pain rating: } 4.8 \text { before vs. } 1.77 \text { after. Patient } \\
\text { perception of improvement after the intervention: } 95 \% \text {. }\end{array}$ \\
\hline Alcobía-Díaz et al., 2017 & $\begin{array}{l}\text { Standardized Constant score: } 36 \text { before } \\
\text { vs. } 81 \text { after. }\end{array}$ & $\begin{array}{c}\text { Differences before vs. after: improved flexion }\left(+15^{\circ} / 5^{\circ}\right) \text { and } \\
\text { abduction }\left(+10^{\circ} / 5^{\circ}\right) \text {, not in rotation }\left(-2^{\circ} / 0^{\circ}\right) \text {. Goutallier } \\
\text { classification: }>2 \text { grade. }\end{array}$ & $\begin{array}{c}\text { Daily activity questionnaire: } 20 \% \text { limitation in shoulder } \\
\text { function with low-demand tasks, } 51 \% \text { limitation with } \\
\text { high-demand tasks. VAS pain }=3.5 \text {. }\end{array}$ \\
\hline Torrens et al., 2016 & $\begin{array}{l}\text { Global score: } 30 \text { before- }-58 \text { at one year. } \\
\text { Pain: } 5 \text { before vs. } 10 \text { at one-year } \\
\text { follow-up. Daily activities: } 8 \text { before vs. } \\
14 \text { at one-year follow-up. }\end{array}$ & $\begin{array}{c}\text { Flexion }=4 \text { before vs. } 7 \text { at one-year follow-up. Abduction = } \\
4 \text { before vs. } 6 \text { at one-year follow-up. External rotation }=3 \\
\text { before vs. } 5 \text { at one-year follow-up. Internal rotation }=4 \\
\text { before vs. } 5 \text { at one-year follow-up }(p=0.1) \text {. Strength }=2 \\
\text { before vs. } 10 \text { at one-year follow-up. }\end{array}$ & $\begin{array}{c}\text { Patient perception of improvement: General }=80 \%, \\
\text { (minimum } 8 \text { points), Strength }=62 \% \text { (minimum } 11 \text { points), } \\
\text { Anterior elevation }=73 \% \text { (minimum } 6 \text { points), Lateral } \\
\text { rotation }=73 \% \text { (minimum } 2 \text { points), Internal rotation }=38 \% \\
\text { (minimum } 2 \text { points). }\end{array}$ \\
\hline Torrens et al., 2017 & $\begin{array}{l}\text { Global score at 2-year follow-up: } 54 . \\
\quad \text { Pain }=12 \text {. Daily activity }=15 .\end{array}$ & $\begin{array}{c}\text { Flexion }=7, \text { Abduction }=6, \text { Lateral rotation }=5, \text { Internal } \\
\text { rotation }=5, \text { Strength }=5 .\end{array}$ & $\begin{array}{l}\text { C-reactive protein; increase after surgery, peak on the 2nd } \\
\text { day, recovery on the 14th. }\end{array}$ \\
\hline Delgado-Rodríguez et al., 2013 & & $\begin{array}{c}\text { Flexion }=39^{\circ} \text { before vs. } 84^{\circ} \text { after. External rotation }=13^{\circ} \\
\text { before vs. } 33^{\circ} \text { after. Internal rotation }=11^{\circ} \text { before vs. } 31^{\circ} \\
\text { after. Abduction }=32^{\circ} \text { before vs. } 75^{\circ} \text { after. }\end{array}$ & QuickDASH score: $36 \%$ after. Pain VAS: 3. \\
\hline Hernández-Elena et al., 2015 & Global score: 63 . Pain:14. & $\begin{array}{c}\text { Abduction }=104^{\circ} . \text { Flexion }=106^{\circ} \text {. Internal rotation }=40^{\circ} . \\
\text { External rotation }=46^{\circ} .\end{array}$ & \\
\hline Villodre-Jiménez et al., 2016 & $\begin{array}{l}\text { Global score: } 65 \text {. Best results in patients } \\
\text { with arm lengthening intervention } \\
<20 \mathrm{~mm} \text {. }\end{array}$ & Flexion $=124^{\circ}$. External rotation $=13^{\circ}$. Abduction $=95^{\circ}$. & $\begin{array}{l}\text { UCLA scale: } 27 \text { points. QuickDASH: } 32 \text {. Best results in } \\
\text { QuickDASH in patients with arm lengthening intervention } \\
<20 \mathrm{~mm} \text {. Patients with moderate to severe pain: } 20 \% \text {. Patients } \\
\text { satisfied: } 95 \% \text {. }\end{array}$ \\
\hline Andrés-Cano et al., 2014 & $\begin{array}{l}\text { Global score at the end of follow-up: } \\
\qquad 4 \text { points. }\end{array}$ & $\begin{array}{l}\text { Active abduction }=50^{\circ} \text {. Flexion }=70^{\circ} \text {. External rotation: } \\
50^{\circ} \text {. Internal rotation: up to the lumbosacral joint. }\end{array}$ & $\begin{array}{c}\text { QuickDASH: } 24 \text { points. Higher number of rehabilitation } \\
\text { sessions = QuickDASH. Less operating time = QuickDASH. } \\
\text { Pain EVA }=1 \text { of } 8 .\end{array}$ \\
\hline
\end{tabular}

36; ASES: American Shoulder and Elbow Surgeons; EQ-5D: Euro QoL 5D; VAS: Visual Analog Scale. 


\section{Discussion}

The results of the review show that shoulder arthroplasty in Spain can currently be considered an effective and safe procedure, with functional recovery and pain reduction in patients operated on for humeral fracture and rotator cuff arthropathy, fracture sequelae and malunion of the proximal humerus, and degenerative diseases. These results are similar to those found in other countries, including Norway, Germany, the Netherlands and the USA [9,15,49-51], with better results observed with reverse-type arthroplasties than hemiarthroplasties.

Regarding the safety of shoulder arthroplasty, the overall complication rate in this procedure appears to be centred around $15 \%$, with the most commonly observed complications being instability, periprosthetic fracture or infection $[13,14]$. The results obtained from this study show that the most frequent complications in Spain were of the same profile as other countries, with similar rates also reported [9,15,52-54]. In addition, increased safety has been observed in recent years worldwide as reported by the Nordic or the Kaiser Permanente registers [15,55]. These improved results could be for various reasons, with progress in prosthesis design being particularly relevant. The presence of reverse prostheses should be noted as they have become one of the treatments of choice for pathologies like proximal humeral fractures or rotator cuff arthropathy [56-59]. The results obtained when assessing reverse prostheses suggest that, while their rate of complications might be slightly higher than those observed in other contexts $[9,15,52-54]$, their results could be better when compared to other types of prostheses $[32,33,36,48]$. This facilitates the hypothesis that reduced incidence of the aforementioned complications could be largely due to an increased use of these types of implants.

Currently, the evidence for implant survival in Spain at the population level is limited. However, the results of the reviewed studies are similar to data from international registries, which estimate an implant revision rate of approximately $90 \%-95 \%$ at 5 and 10 years $[9,15,49,50,52]$. In addition, most of the prosthesis models identified in this review are commonly used internationally, and the ODEP assessment of most of those included in the selected studies was acceptable [9,25]. Similar to our results, the 10-year cumulative revision rate after primary reverse shoulder arthroplasty in the Nordic countries was between $90 \%$ to $95 \%$ and the model most frequently used was Delta Xtend [55]. Moreover, the results from other European shoulder arthroplasty registers, including the National Joint Registry (NJR) in the United Kingdom and the Dutch arthroplasty register (LROI) show that the results in terms of survival rates might be similar across European countries and that the prosthesis models most frequently used are usually the same [51,60]. However, considering the results for shoulder arthroplasty effectiveness, in terms of functionality, pain and impact on the patient's life, there is some difference in calculating scores, which hinders synthesis [61,62]. Regardless of the calculation differences, especially for the Constant-Murley test, improved scores after the intervention were observed in the results of the studies reviewed. The results show that an improvement in pain could be relevant, even at short-term follow-ups after arthroplasty, which is contrary to results suggested by a previous study proposing that improvement in the short-term may not be as evident as in longer-term follow-ups [63].

The authors accept that a significant limitation of the present study is the search strategy. Given its focus on the Spanish population, extrapolating and generalizing its results to other populations is challenging. However, we believe that delimiting the safety and effectiveness of shoulder arthroplasties in a specific healthcare context may be useful in encouraging and improving results at all levels: surgical, management, and patient. In addition, the results shown can be useful and relevant at the international level when making comparisons and establishing common standards of reference. It is also important to mention the limitation related to the inclusion criteria, given that patients under 18 years old and patients with tumours were excluded. These criteria restrict the capacity to extrapolate the results obtained to the whole population. Despite this limitation, the results found could be applicable to most of patients eligible for a shoulder arthroplasty, and thus it is reasonable to assume that they could be at least approaching the true results of these procedures in Spain. Another limitation is related to the heterogeneity of the studies in terms of their design and presentation of 
results, which makes a meta-analysis impossible. However, the evidence presented was synthesized as much as possible to be able to serve both as a reference in assessing the safety and effectiveness of shoulder arthroplasty, and as a starting point for new studies on the subject. Lastly, these studies were considered without stratifying by patient diagnosis or the severity of their symptoms. As such, it is possible that some of the results described correspond to selected samples of patients with certain diagnoses. Despite this, most of the studies focus on the two main reasons for intervention in shoulder arthroplasty: fracture of the humerus and rotator cuff arthropathy, which is why we believe the results shown can be widely generalized to the population susceptible to receiving a shoulder arthroplasty and not only to those with one of the two diagnoses. However, further epidemiological research stratified by these indications both in Spain and in other countries could be valuable to obtain a more precise representation of the safety and effectiveness of shoulder arthroplasties at the population level.

\section{Conclusions}

In Spain, primary shoulder arthroplasties, for those who are able to receive them, are an effective and safe procedure that allow functional recovery and pain reduction in patients with humeral fracture, rotator cuff arthropathy, fracture sequelae and malunion of the proximal humerus, and degenerative diseases. The prosthesis type with the best survival is the reverse prosthesis. Future longitudinal population-based studies, particularly randomized controlled trials, as well as the establishment of a shoulder arthroplasty registry could confirm these results and identify areas of improvement, including the recommendation of specific types of prostheses or models with preferable results.

Author Contributions: All authors conceived the study design, participated in writing the manuscript, and have critically reviewed and agreed on the final version of this article.

Funding: This study was funded by the partnership agreement signed by the Carlos III Health Institute, an autonomous body of the Ministry of Economy and Competitiveness, and the Agency for Health Quality and Assessment of Catalonia, within the activity development framework of the Spanish Network of Health Technology and Performance Evaluation Agencies of the National Health System, funded by the Ministry of Health, Social Services and Equality.

Acknowledgments: We acknowledge Carlos III Health Institute, CIBER Epidemiology and Public Health (CIBERESP), Antoni Parada and Olga Martinez for their support.

Conflicts of Interest: The authors declare no conflict of interest.

\section{Appendix A PubMed Filter}

\#1 shoulder[MeSH] OR shoulder[ti] OR humeral[ti] OR glenohumeral[ti] OR glenoid[ti]

\#2 arthroplasty[MeSH] OR arthroplast*[ti] OR replacement[ti] OR "prosthesis implantation"

[MeSH] OR prosthes*[ti] OR implant* OR "prostheses and implants"[MeSH]

\#3 Spain[tiab] OR Spanish[tiab] OR España[tiab] OR Espana[tiab] OR Espan*[tiab] OR Andalusia*[tiab] OR Catalan*[tiab] OR Catalonian[tiab] OR Madrid[tiab] OR Madrilen*[tiab] OR Madrileñ*[tiab] OR Valencian*[tiab] OR Galicia[tiab] OR Galego[tiab] OR Galleg*[tiab] OR Galaic[tiab] OR “Castilla y Leon"[tiab] OR “Castilla and Leon"[tiab] OR Leones*[tiab] OR Basque[tiab] OR basc*[tiab] OR "Castilla-La Mancha"[tiab] OR Canary[tiab] OR Canarian[tiab] OR Canari*[tiab] OR Murcia[tiab] OR Murcian*[tiab] OR Aragon*[tiab] OR Extremadura[tiab] OR Extremeno[tiab] OR Extremena[tiab] OR Extremeño[tiab] OR Extremeña[tiab] OR Balear[tiab] OR Asturias[tiab] OR Asturian*[tiab] OR Navarra[tiab] OR Navarre[tiab] OR Cantabria[tiab] OR Cantabric*[tiab] OR “La Rioja"[tiab] OR Riojan*[tiab]

\#4 spain[MeSH] OR spain OR espagne OR espana OR spain[ad] OR espagne[ad] OR espana[ad] OR osasunbidea[ad] OR osakidetza[ad] OR insalud[ad] OR sergas[ad] OR catalunya[ad] OR catalonia[ad] OR catalogne[ad] OR cataluna[ad] OR catala[ad] OR barcelon[ad] OR barcelona[ad] OR barcelones[ad] OR barceloneta[ad] OR barcelonia[ad] OR tarragona[ad] OR lleida[ad] OR lerida[ad] OR girona[ad] OR gerona[ad] OR sabadell[ad] OR hospitalet[ad] OR l'hospitalet[ad] OR valencia[ad] OR castello[ad] OR castellon[ad] OR alacant[ad] OR alicant[ad] OR alicante[ad] OR murcia[ad] OR murcian OR 
murciana[ad] OR murciano[ad] OR andaluci[ad] OR andalucia[ad] OR andaluciajunta[ad] OR andalusi[ad] OR andalusia[ad] OR andalusian[ad] OR andaluz[ad] OR andaluza[ad] OR sevill[ad] OR sevilla[ad] OR seville[ad] OR granada[ad] OR huelva[ad] OR almeria[ad] OR cadiz[ad] OR jaen[ad] OR malaga[ad] OR extremadura[ad] OR caceres[ad] OR badajoz[ad] OR madrid[ad] OR galicia[ad] OR gallego[ad] OR compostela[ad] OR vigo[ad] OR coruna[ad] OR ferrol[ad] OR orense[ad] OR ourense[ad] OR pontevedra[ad] OR oviedo[ad] OR gijon[ad] OR asturia[ad] OR asturiano[ad] OR asturias[ad] OR asturias[ad] OR cantabria[ad] OR cantabrico[ad] OR cantabro[ad] OR santander[ad] OR vasco[ad] OR euskadi[ad] OR basque[ad] OR bilbao[ad] OR bilbo[ad] OR donosti[ad] OR donostia[ad] OR vizcaya[ad] OR guipuzcoa[ad] OR gipuzkoa[ad] OR alava[ad] OR alaba[ad] OR vitoria[ad] OR vitoria[ad] OR vitoria-gasteiz[ad] OR bizkaia[ad] OR navarra[ad] OR pamplona[ad] OR irunea[ad] OR aragon[ad] OR aragones[ad] OR zaragoza[ad] OR teruel[ad] OR huesca[ad] OR mancha[ad] OR "ciudad real"[ad] OR albacete[ad] OR cuenca[ad] OR balear[ad] OR baleares[ad] OR balearic[ad] OR balears[ad] OR mallorca[ad] OR menorca[ad] OR ibiza[ad] OR eivissa[ad] OR palmas[ad] OR lanzarote[ad] OR canaria[ad] OR canarian[ad] OR canarias[ad] OR canario[ad] OR tenerife[ad] OR castilla[ad] OR salamanca[ad] OR zamora[ad] OR valladolid[ad] OR segovia[ad] OR soria[ad] OR palencia[ad] OR avila[ad] OR burgos[ad] OR (leon[ad] NOT (france[ad] OR clermont[ad] OR rennes[ad] OR lyon[ad] OR USA[ad] OR mexic[ad] OR mexica[ad])) OR (cordoba[ad] NOT (argentin[ad] OR argentina[ad])) OR (toledo[ad] NOT (ohio[ad] OR us[ad] OR usa[ad] OR OH[ad])) OR (guadalajara[ad] NOT (mexic[ad] OR mexica[ad] OR mexicali[ad] OR mexican[ad] OR mexicana[ad] OR mexicano[ad] OR mexicanos[ad]))

\#5 \#1 AND \#2 AND (\#3 OR \#4) Filters: Publication date from 2003/01/01

\section{References}

1. Boileau, P.; Sinnerton, R.J.; Chuinard, C.; Walch, G. Arthroplasty of the shoulder. J. Bone Jt. Surg. Br. 2006, 88, 562-575. [CrossRef] [PubMed]

2. Rangan, A.; Handoll, H.; Brealey, S.; Jefferson, L.; Keding, A.; Martin, B.; Goodchild, L.; Chuang, L.; Hewitt, C.; Torgerson, D. Surgical vs. nonsurgical treatment of adults with displaced fractures of the proximal humerus. Ned. Tijdschr. Traumachir. 2016, 24, 18. [CrossRef]

3. Handoll, H.; Brealey, S.; Rangan, A.; Keding, A.; Corbacho, B.; Jefferson, L.; Chuang, L.-H.; Goodchild, L.; Hewitt, C.; Torgerson, D. The ProFHer (PROximal fracture of the humerus: Evaluation by randomisation) trial-A pragmatic multicentre randomized controlled trial evaluating the clinical effectiveness and cost-effectiveness of surgical compared with non-surgical treatment for proxi. Health Technol. Assess. 2015, 19, 1-279. [CrossRef] [PubMed]

4. Singh, J.A.; Sperling, J.; Buchbinder, R.; McMaken, K. Surgery for shoulder osteoarthritis. Cochrane Database Syst. Rev. 2010, 6, CD008089. [CrossRef] [PubMed]

5. Sumrein, B.O.; Huttunen, T.T.; Launonen, A.P.; Berg, H.E.; Fellander-Tsai, L.; Mattila, V.M. Proximal humeral fractures in Sweden-A registry-based study. Osteoporos. Int. 2017, 28, 901-907. [CrossRef] [PubMed]

6. Ryösä, A.; Laimi, K.; Äärimaa, V.; Lehtimäki, K.; Kukkonen, J.; Saltychev, M. Surgery or conservative treatment for rotator cuff tear: A meta-analysis. Disabil. Rehabil. 2017, 39, 1357-1363. [CrossRef]

7. Sharma, S.; Dreghorn, C.R. Registry of shoulder arthroplasty-The Scottish experience. Ann. R. Coll. Surg. Engl. 2006, 88, 122-126. [CrossRef]

8. Handoll, H.H.; Brorson, S. Interventions for treating proximal humeral fractures in adults. Cochrane Database Syst. Rev. 2015, 11, CD000434. [CrossRef]

9. Lubbeke, A.; Rees, J.L.; Barea, C.; Combescure, C.; Carr, A.J.; Silman, A.J. International variation in shoulder arthroplasty. Acta Orthop. 2017, 88, 592-599. [CrossRef]

10. Arias-de la Torre, J.; Capdevila, A.; Martínez, O.; Domingo, L.; Marinelli, M.; Robles, N.; Nardi, J.; Puig-Verdié, L.; Pallisó, F.; Espallargues, M.; et al. A decade of the Catalonian Arthroplasty Register (RACat): Variability, exhaustivity, and survival of prostheses between 2005 and 2014. Rev. Esp. Cir. Ortop. Traumatol. 2017, 61, 70-81. [CrossRef]

11. Portal estadístico Ministerio de Sanidad, Servicios Sociales e Igualdad Conjunto Mínimo Básico de Datos al Alta Hospitalria. 
12. Familiari, F.; Rojas, J.; Doral, M.N.; Huri, G.; McFarland, E.G. Reverse total shoulder arthroplasty. EFORT Open Rev. 2018. [CrossRef] [PubMed]

13. Gauci, M.-O.; Cavalier, M.; Gonzalez, J.-F.; Holzer, N.; Baring, T.; Walch, G.; Boileau, P. Revision of failed shoulder arthroplasty: Epidemiology, etiology, and surgical options. J. Shoulder Elb. Surg. 2019. [CrossRef] [PubMed]

14. Bohsali, K.I.; Bois, A.J.; Wirth, M.A. Complications of shoulder arthroplasty. J. Bone Jt. Surg. Am. 2017, 1, 256-269. [CrossRef] [PubMed]

15. Dillon, M.T.; Ake, C.F.; Burke, M.F.; Singh, A.; Yian, E.H.; Paxton, E.W.; Navarro, R.A. The kaiser permanente shoulder arthroplasty registry: Results from 6336 primary shoulder arthroplasties. Acta Orthop. 2015, 86, 286-292. [CrossRef]

16. Australian Orthopaedic Association National Joint Replacement Registry (AOANJRR). Annual Report 2016; AOANJRR: Adelaide, Australia, 2016.

17. Hernandez, N.M.; Chalmers, B.P.; Wagner, E.R.; Sperling, J.W.; Cofield, R.H.; Sanchez-Sotelo, J. Revision to reverse total shoulder arthroplasty restores stability for patients with unstable shoulder prostheses. Clin. Orthop. Relat. Res. 2017, 475, 2716-2722. [CrossRef]

18. Alcobia-Diaz, B.; Lopiz, Y.; Garcia-Fernandez, C.; Rizo de Alvaro, B.; Marco, F. Patient reported activities after reverse total shoulder arthroplasty in rotator cuff arthropathy patients. Rev. Esp. Cir. Ortop. Traumatol. 2017, 61, 273-280. [CrossRef]

19. Andrés-Cano, P.; Galán, A.; Arenas, J.; Del Águila, B.; Guerado, E. Results of uncemented hemiarthroplasty as primary treatment of severe proximal humerus fractures in the elderly. Eur. J. Orthop. Surg. Traumatol. 2014, 25, 273-280. [CrossRef]

20. Martinez, A.A.; Calvo, A.; Bejarano, C.; Carbonel, I.; Herrera, A. The use of the Lima reverse shoulder arthroplasty for the treatment of fracture sequelae of the proximal humerus. J. Orthop. Sci. 2012, 17, 141-147. [CrossRef]

21. Liberati, A.; Altman, D.G.; Tetzlaff, J.; Mulrow, C.; Gøtzsche, P.C.; Ioannidis, J.P.A.; Clarke, M.; Devereaux, P.J.; Kleijnen, J.; Moher, D. The PRISMA statement for reporting systematic reviews and meta-analyses of studies that evaluate health care interventions: Explanation and elaboration. J. Clin. Epidemiol. 2009, 62, e1-e34. [CrossRef]

22. Arias-de la Torre, J.; Puigdomenech, E.; Valderas, J.M.; Evans, J.P.; Martín, V.; Molina, A.J.; Rodríguez, N.; Espallargues, M. Availability of specific tools to assess patient reported outcomes in hip arthroplasty in Spain. Identifying the best candidates to incorporate in an arthroplasty register: A systematic review and standardized assessment. PLoS ONE 2019, 14, e0214746. [CrossRef]

23. Valderas, J.M.; Mendivil, J.; Parada, A.; Losada-Yáñez, M.; Alonso, J. Construcción de un filtro geográfico para la identificación en PubMed de estudios realizados en España. Rev. Española Cardiol. 2006, 59, 1244-1251. [CrossRef]

24. Terwee, C.B.; Jansma, E.P.; Riphagen, I.I.; de Vet, H.C.W. Development of a methodological PubMed search filter for finding studies on measurement properties of measurement instruments. Qual. Life Res. 2009, 18, 1115-1123. [CrossRef] [PubMed]

25. Northgate Orthopaedic Data Evaluation Panel (ODEP). Available online: http://www.odep.org.uk/ (accessed on 8 October 2019).

26. Harbour, R.; Miller, J. A new system for grading recommendations in evidence based guidelines. BMJ 2002, 323, 334-336. [CrossRef] [PubMed]

27. Higgins, J.P.T.; Savović, J.; Page, M.J.; Sterne, J.A.C. Revised Cochrane Risk of Bias Tool for Randomized Trials (RoB 2.0). Available online: https:/www.bristol.ac.uk/media-library/sites/social-community-medicine/ images/centres/cresyda/RoB2-0_indiv_main_guidance.pdf (accessed on 8 October 2019).

28. Sterne, J.A.; Hernán, M.A.; Reeves, B.C.; Savović, J.; Berkman, N.D.; Viswanathan, M.; Henry, D.; Altman, D.G.; Ansari, M.T.; Boutron, I.; et al. ROBINS-I: A tool for assessing risk of bias in non-randomised studies of interventions. BMJ 2016. [CrossRef] [PubMed]

29. Moga, C.; Guo, B.; Harstall, C. Development of a Quality Appraisal Tool for Case Series Studies Using a Modified Delphi Technique; Institute of Health Economics: Edmonton, AB, Canada, 2012.

30. Torrens, C.; Guirro, P.; Santana, F. The minimal clinically important difference for function and strength in patients undergoing reverse shoulder arthroplasty. J. Shoulder Elb. Surg. 2016, 25, 262-268. [CrossRef] [PubMed] 
31. Torrens, C.; Guirro, P.; Miquel, J.; Santana, F. Influence of glenosphere size on the development of scapular notching: A prospective randomized study. J. Shoulder Elb. Surg. 2016, 25, 1735-1741. [CrossRef] [PubMed]

32. Sebastia-Forcada, E.; Cebrian-Gomez, R.; Lizaur-Utrila, A.; Gil-Guillen, V. Reverse shoulder arthroplasty versus hemiarthroplasty for acute proximal humeral fractures: A blinded, randomized, controlled, prospective study. J. Shoulder Elb. Surg. 2014, 23, 1419-1426. [CrossRef]

33. Boyer, E.; Menu, G.; Loisel, F.; Saadnia, R.; Uhring, J.; Adam, A.; Rochet, S.; Clappaz, P.; Baudouin, E.; Lascar, T.; et al. Cementless and locked prosthesis for the treatment of 3-part and 4-part proximal humerus fractures: Prospective clinical evaluation of hemi- and reverse arthroplasty. Eur. J. Orthop. Surg. Traumatol. 2017, 27, 301-308. [CrossRef]

34. Alentorn-Geli, E.; Guirro, P.; Santana, F.; Torrens, C. Treatment of fracture sequelae of the proximal humerus: Comparison of hemiarthroplasty and reverse total shoulder arthroplasty. Arch. Orthop. Trauma Surg. 2014, 134, 1545-1550. [CrossRef]

35. Jorge-Mora, A.; Amhaz-Escanlar, S.; Fernández-Pose, S.; Lope-Del-Teso, C.; Pino-Mínguez, J.; Caeiro-Rey, J.R.; Pretell-Mazzini, J.; Gómez, R. Early outcomes of locked noncemented stems for the management of proximal humeral fractures: A comparative study. J. Shoulder Elb. Surg. 2019, 28, 48-55. [CrossRef]

36. Sebastia-Forcada, E.; Lizaur-Utrilla, A.; Cebrian-Gomez, R.; Miralles-Muñoz, F.A.; Lopez-Prats, F.A. Outcomes of reverse total shoulder arthroplasty for proximal humeral fractures: Primary arthroplasty versus secondary arthroplasty after failed proximal humeral locking plate fixation. J. Orthop. Trauma 2017, 31, e236-e240. [CrossRef] [PubMed]

37. Lopiz, Y.; García-Coiradas, J.; Serrano-Mateo, L.; García-Fernández, C.; Marco, F. Reverse shoulder arthroplasty for acute proximal humeral fractures in the geriatric patient: Results, health-related quality of life and complication rates. Int. Orthop. 2016, 40, 771-781. [CrossRef] [PubMed]

38. Bonilla, J.C. Pròtesi Invertida d'Espatlla: Estudi Retrospectiu del Conflicte Escapular en 2 Models Diferents de Pròtesis; Universitat Autònoma de Barcelona, Departament de Cirurgia: Barcelona, Spain, 2012.

39. Izquierdo-Fernández, A.; Minarro, J.C.; Carpintero-Lluch, R.; Estévez-Torres, E.M.; Carpintero-Benítez, P. Reverse shoulder arthroplasty in obese patients: Analysis of functionality in the medium-term. Arch. Orthop. Trauma Surg. 2017, 138, 5. [CrossRef] [PubMed]

40. Cáceres-Sánchez, L.; Mesa-Mateo, A.; Barrionuevo-Sánchez, F.J.; García-Benítez, B.; Expósito-Triano, S. Total reverse shoulder replacement: Evaluation of the clinical results and complications in a series of 52 cases. Rev. Esp. Cir. Ortop. Traumatol. 2015, 59, 439-446. [CrossRef] [PubMed]

41. Zafra, M.; Uceda, P.; Flores, M.; Carpintero, P. Reverse total shoulder replacement for nonunion of a fracture of the proximal humerus. Bone Jt. J. 2014, 96, 1239-1243. [CrossRef] [PubMed]

42. García-Fernández, C.; Lópiz-Morales, Y.; Rodríguez, A.; López-Durán, L.; Martínez, F.M. Periprosthetic humeral fractures associated with reverse total shoulder arthroplasty: Incidence and management. Int. Orthop. 2015, 39, 1965-1969. [CrossRef]

43. Torrens, C.; Santana, F.; Marí, R.; Puig, L.; Alier, A. Serum C-reactive protein in patients undergoing elective shoulder arthroplasty: Prospective study. J. Orthop. Sci. 2017, 22, 858-861. [CrossRef]

44. Torrens, C.; Alentorn-Geli, E.; Mingo, F.; Gamba, C.; Santana, F. Reverse shoulder arthroplasty for the treatment of acute complex proximal humeral fractures: Influence of greater tuberosity healing on the functional outcomes. J. Orthop. Surg. 2018, 26, 2309499018760132. [CrossRef]

45. Delgado Rodriguez, J.A.; Moreno Palacios, J.A.; Pulido Poma, R.M.; Fernandez Leon, R.A.; Martin Maroto, M.P.; Miranda Vivas, M.T. Functional results of partial shoulder replacement in patients over 65 years. Rev. Esp. Geriatr. Gerontol. 2013, 48, 22-25.

46. Hernández-Elena, J.; de la Red-Gallego, M.Á.; Garcés-Zarzalejo, C.; Pascual-Carra, M.A.; Pérez-Aguilar, M.D.; Rodríguez-López, T.; Alfonso-Fernández, A.; Pérez-Núñez, M.I. Treatment of proximal humeral fractures by reverse shoulder arthroplasty: Mid-Term evaluation of functional results and Notching. Rev. Esp. Cir. Ortop. Traumatol. 2015, 59, 413-420. [CrossRef]

47. Villodre-Jiménez, J.; Estrems-Díaz, V.; Diranzo-García, J.; Bru-Pomer, A. Reverse shoulder arthroplasty in 3 and 4 part proximal humeral fractures in patients aged more than 65 years: Results and complications. Rev. Esp. Cir. Ortop. Traumatol. 2016, 61, 43-50. [CrossRef] [PubMed]

48. Sebastià-Forcada, E. Influencia de los Factores Anatómicos en el Resultado de las Prótesis de Hombro para el Tratamiento de las Fracturas Proximales de Húmero en Pacientes de Edad. 2014. Available online: https://dialnet.unirioja.es/servlet/cittes?codigo=68217 (accessed on 8 October 2019). 
49. Blomquist, J.; Solheim, E.; Liavaag, S.; Schroder, C.P.; Espehaug, B.; Havelin, L.I. Shoulder instability surgery in Norway: The first report from a multicenter register, with 1-year follow-up. Acta Orthop. 2012, 83, 165-170. [CrossRef] [PubMed]

50. Magosch, P.; Habermeyer, P.; Lichtenberg, S.; Tauber, M.; Gohlke, F.; Mauch, F.; Boehm, D.; Loew, M.; Zeifang, F.; Potzl, W. Results from the German shoulder- and elbow-arthroplasty register (SEPR): Anatomic or reverse shoulder arthroplasty in B2-glenoids? Orthopade 2017, 46, 1063-1072. [CrossRef] [PubMed]

51. Dutch Arthroplasty Register (LROI). Annual Repport, 2018; LROI: Hague, The Netherlands, 2018.

52. Fevang, B.-T.S.; Lie, S.A.; Havelin, L.I.; Skredderstuen, A.; Furnes, O. Risk factors for revision after shoulder arthroplasty: 1825 shoulder arthroplasties from the Norwegian Arthroplasty Register. Acta Orthop. 2009, 80, 83-91. [CrossRef] [PubMed]

53. Rasmussen, J.V.; Olsen, B.S.; Fevang, B.-T.S.; Furnes, O.; Skytta, E.T.; Rahme, H.; Salomonsson, B.; Mohammed, K.D.; Page, R.S.; Carr, A.J. A review of national shoulder and elbow joint replacement registries. J. Shoulder Elb. Surg. 2012, 21, 1328-1335. [CrossRef]

54. Alentorn-Geli, E.; Clark, N.J.; Assenmacher, A.T.; Samuelsen, B.T.; Sanchez-Sotelo, J.; Cofield, R.H.; Sperling, J.W. What are the complications, survival, and outcomes after revision to reverse shoulder arthroplasty in patients older than 80 years? Clin. Orthop. Relat. Res. 2017, 475, 2744-2751. [CrossRef]

55. Lehtimaki, K.; Rasmussen, J.V.; Mokka, J.; Salomonsson, B.; Hole, R.; Jensen, S.L.; Aarimaa, V. Risk and risk factors for revision after primary reverse shoulder arthroplasty for cuff tear arthropathy and osteoarthritis: A Nordic Arthroplasty Register Association study. J. Shoulder Elb. Surg. 2018, 27, 1596-1601. [CrossRef]

56. Erickson, B.J.; Bohl, D.D.; Cole, B.J.; Verma, N.N.; Nicholson, G.; Romeo, A.A.; Harris, J.D. Reverse total shoulder arthroplasty: Indications and techniques across the world. Am. J. Orthop. 2018, 47. [CrossRef]

57. Drake, G.N.; O'Connor, D.P.; Edwards, T.B. Indications for reverse total shoulder arthroplasty in rotator cuff disease. Clin. Orthop. Relat. Res. 2010, 468, 1526-1533. [CrossRef]

58. Nolan, B.M.; Ankerson, E.; Wiater, J.M. Reverse total shoulder arthroplasty improves function in cuff tear arthropathy. Clin. Orthop. Relat. Res. 2011, 469, 2476-2482. [CrossRef]

59. Jobin, C.M.; Galdi, B.; Anakwenze, O.A.; Ahmad, C.S.; Levine, W.N. Reverse shoulder arthroplasty for the management of proximal humerus fractures. J. Am. Acad. Orthop. Surg. 2015, 23, 190-201. [CrossRef] [PubMed]

60. National Joint Registry for England, Wales; Northern Ireland and the Isle of Man (NJR). UK National Jt. Registry 16th Annual Report; NJR: London, UK, 2018.

61. Angst, F.; Schwyzer, H.-K.; Aeschlimann, A.; Simmen, B.R.; Goldhahn, J. Measures of adult shoulder function: Disabilities of the arm, shoulder, and hand questionnaire (DASH) and its short version (QuickDASH), Shoulder Pain and Disability Index (SPADI), American Shoulder and Elbow Surgeons (ASES) Society standardized shoulder. Arthritis Care Res. 2011, 63, S174-S188. [CrossRef] [PubMed]

62. Constant, C.R. An evaluation of the constant-murley shoulder assessment. J. Bone Jt. Surg. Br. 1997, 79, 695-696. [CrossRef]

63. Bjornholdt, K.T.; Brandsborg, B.; Soballe, K.; Nikolajsen, L. Persistent pain is common 1-2 years after shoulder replacement. Acta Orthop. 2015, 86, 71-77. [CrossRef] [PubMed]

(C) 2019 by the authors. Licensee MDPI, Basel, Switzerland. This article is an open access article distributed under the terms and conditions of the Creative Commons Attribution (CC BY) license (http://creativecommons.org/licenses/by/4.0/). 